Original paper

\title{
Proton microbeam radiotherapy with scanned pencil-beams - Monte Carlo simulations
}

\author{
M. Kłodowska ${ }^{\text {a, * }}$, P. Olko a , M.P.R. Waligórski ${ }^{\text {a, b }}$ \\ a Institute of Nuclear Physics Polish Academy of Sciences, Radzikowskiego 152, PL 31-342 Kraków, Poland \\ ${ }^{\mathrm{b}}$ The Marie-Skłodowska-Curie Centre of Oncology, Kraków Division, Kraków, Poland
}

\section{A R T I C L E I N F O}

Article history:

Received 20 December 2014

Received in revised form

9 April 2015

Accepted 11 April 2015

Available online 15 May 2015

Keywords:

Microbeam radiation therapy

Proton radiotherapy

Monte Carlo simulations

\begin{abstract}
A B S T R A C T
Irradiation, delivered by a synchrotron facility, using a set of highly collimated, narrow and parallel photon beams spaced by $1 \mathrm{~mm}$ or less, has been termed Microbeam Radiation Therapy (MRT). The tolerance of healthy tissue after MRT was found to be better than after standard broad X-ray beams, together with a more pronounced response of malignant tissue. The microbeam spacing and transverse peak-to-valley dose ratio (PVDR) are considered to be relevant biological MRT parameters. We investigated the MRT concept for proton microbeams, where we expected different depth-dose profiles and PVDR dependences, resulting in skin sparing and homogeneous dose distributions at larger beam depths, due to differences between interactions of proton and photon beams in tissue. Using the FLUKA Monte Carlo code we simulated PVDR distributions for differently spaced $0.1 \mathrm{~mm}$ (sigma) pencil-beams of entrance energies $60,80,100$ and $120 \mathrm{MeV}$ irradiating a cylindrical water phantom with and without a bone layer, representing human head. We calculated PVDR distributions and evaluated uniformity of target irradiation at distal beam ranges of $60-120 \mathrm{MeV}$ microbeams. We also calculated PVDR distributions for a $60 \mathrm{MeV}$ spread-out Bragg peak microbeam configuration. Application of optimised proton MRT in terms of spot size, pencil-beam distribution, entrance beam energy, multiport irradiation, combined with relevant radiobiological investigations, could pave the way for hypofractionation scenarios where tissue sparing at the entrance, better malignant tissue response and better dose conformity of target volume irradiation could be achieved, compared with present proton beam radiotherapy configurations.

(C) 2015 Published by Elsevier Ltd on behalf of Associazione Italiana di Fisica Medica. This is an open access article under the CC BY-NC-ND license (http://creativecommons.org/licenses/by-nc-nd/4.0/).
\end{abstract}

\section{Introduction}

The principal aim of radiotherapy is to uniformly irradiate the target volume with the recommended dose while sparing the adjacent healthy tissues and critical organs. In microbeam radiation therapy (MRT), the tumour is irradiated with closely spaced narrow photon microbeams of high intensity which locally deliver a high dose to the tissue [1]. The biological advantages of this unique technique appear to stem from better regeneration of the microvascular structure of the irradiated healthy tissues outside the direct paths of the microbeams (i.e. within the "dose valleys" between them) and from the dose-volume effect, both of which improve the healthy versus malignant tissue response $[1,2]$. The microbeam spacing and the peak-to-valley-dose-ratio (PVDR) are

\footnotetext{
* Corresponding author.

E-mail address: Magdalena.Klodowska@ifj.edu.pl (M. Kłodowska).
}

believed to be important factors in this technique. With high enough PVDR values, tissue recovery at local doses up to several hundred Gy was achieved, offering a possibility of better local tumour control in comparison with conventional broad photon beam techniques $[1,2]$.

An alternative technique to conventional MV X-ray radiotherapy is that performed with beams of protons or carbon ions. Charged particles, apart from distal dose enhancement due to the Bragg Peak (BP), have a well-defined energy-dependent range. The latter allows one to considerably limit the absorbed dose to healthy tissues and to critical structures located behind the target. By now, some 40 particle therapy centres operate worldwide, and over 120,000 patients have been treated. Active beam scanning is today's state of the art in proton therapy. Intensity Modulated Proton Radiotherapy (IMPT) using active beam scanning enables the best possible coverage of target volume to be achieved, minimizing the dose to healthy tissue, including dose to the skin. Here, a narrow proton beam is scanned over each plane transverse to the central 
beam axis, over gradually decreasing depths (as controlled by varying the entrance energy of the beam) to cover the target volume. The beam may be delivered from different gantry angles, and inverse-planned to realise an individually optimised treatment plan whereby the required beam spot scanning sequence is implemented by suitably selecting ion fluences and their entrance energies at different gantry angles, to achieve the required dose distribution over the target volume.

The MRT principle, originally developed for photon microbeams can also be applied using narrowly collimated and closely spaced proton beams [3,4]. Zlobinskaya et al. [4] proposed microchannel diameters ranging between $50 \mu \mathrm{m}$ and $500 \mu \mathrm{m}$ and suggested the distance between the channels (point-to-point distance, $d$ ) to be at least 10 times larger than the microchannel radius. The proton pencil microbeam approach could then potentially offer the advantage of higher tissue tolerance, combined with well-defined proton ranges closely tailored to the tumour geometry. They next demonstrated the potential effect of healthy tissue sparing at skin depth in an experiment using $20 \mathrm{MeV}$ protons of the Munich Superconducting Nanoscope for Applied nuclear (Kern-) physics Experiments SNAKE, where the beam was focused to a beam spot diameter of $500 \mathrm{~nm}$ (FWHM), or smaller [5]. Cultured EpiDermFT skin was irradiated with a square channel pattern of $10 \times 10 \mu \mathrm{m}^{2}$ or $50 \times 50 \mu \mathrm{m}^{2}$ to an average dose of $2 \mathrm{~Gy}$ (with local dose values of some $5000 \mathrm{~Gy}$ and $200 \mathrm{~Gy}$, respectively), producing a significantly decreased skin inflammation response compared to a homogeneous $2 \mathrm{~Gy}$ broad beam proton irradiation of a similar skin sample [4]. Following the experience with X-ray microbeams, the importance of peak-to-valley-dose-ratio (PVDR) in proton microbeam irradiation was also stated $[3,4]$.

Unlike in X-ray MRT, due to proton scattering, the adjoining micro-beams will blur with increasing beam depths, possibly leading to a laterally uniform dose distribution delivered to the target at Bragg peak (BP) depths. Thus, the beam energy- and microchannel spacing-dependent PVDR distributions need to be carefully studied to best exploit the potential advantages of proton microbeams, which consist in sparing tissues (skin or bone) at beam entry (related to high PVDR values) and at the same time achieving homogeneous irradiation at target (BP) depths. This is the main conceptual difference between proton and X-ray MRT, where parallel microbeams merely transverse the target volume. A calculation by Zlobinskaya et al. [4], who used the CERR open-source planning system and a semi-empirical proton dose algorithm, demonstrated qualitatively that the concept of proton microchannel irradiation is realistic. As shown by Prezado and Fois [3], Monte Carlo methods, commonly used in particle therapy, e.g. for therapy planning and dose verification, can be employed to investigate the proton microbeam concept.

The general aim of this study was to investigate quantitatively whether it would indeed be possible to achieve a uniform transverse dose distribution over the target volume in a realistic proton microbeam irradiation set up and, at the same time, exploit its advantage in sparing healthy tissues (including, e.g. the skin or skull bone) at beam entry. We applied the FLUKA Monte Carlo transport code [6,7] to study the variation of PVDR between their maximum values at the entrance region and their decrease at greater depths for a proton beam, while achieving a uniform dose distribution at target depths (up to $11 \mathrm{~cm}$ in water), for different microchannel distances and microbeam energies. We selected realistic microbeam parameters (in terms of size and distances) for initial proton energies ranging between $60 \mathrm{MeV}$ and $120 \mathrm{MeV}$ and also simulated a spread-out Bragg peak (SOBP) configuration for a $60 \mathrm{MeV}$ proton beam, to verify the selected beam parameters. We studied the PVDR and depth-dose distributions in a cylindrical water phantom representing the human head and the homogeneity of irradiation of a small target volume inside the phantom. Additional bone layer was also simulated as an outer phantom layer to examine its impact on PVDR distributions. While our study using the FLUKA transport code was limited to the analysis of the dosimetry aspects of proton microbeam radiotherapy (basically via PVDR distributions and homogeneity of target irradiation), we believe it to be also relevant to the radiobiology issues of proton MRT.

\section{Methods}

The FLUKA Monte Carlo (MC) multi-particle transport code ver. 2011.2 has been widely used in particle therapy for dose calculations, plan verification and detector response simulations [7]. To perform sufficiently accurate micro-scale calculations, we set the parameters in the hadron therapy version of this code to limit particle transport to $100 \mathrm{keV}$, to follow neutron transport down to thermal energies, scattered photons down to $1 \mathrm{keV}$, and set the delta ray production threshold at $100 \mathrm{keV}$. All calculations were run in parallel mode, to at least $10^{5}$ protons per spot, for the uncertainty level not to exceed $2 \%$.

The FLUKA SOURCE routine was adjusted to follow the active beam scanning method for proton delivery. A pencil beam with a Gaussian transverse profile of $100 \mu \mathrm{m}$ (1 sigma) was set up. Following the X-ray microbeam experience [2], we selected this beam parameter as being not too unrealistic in any future technical implementation and not too large to affect the healthy tissue locally.

To simulate the geometry of the human head, a cylindrical phantom composed of liquid water was defined, of $16 \mathrm{~cm}$ length and a diameter of $16 \mathrm{~cm}$ [8]. Monte Carlo simulations were performed for $100 \mu \mathrm{m}$ proton microbeams of initial energies of 60,80 , 100 and $120 \mathrm{MeV}$, each with a 1\% full-width-half-maximum (FWHM) Gaussian energy spread. The lateral centre-to-centre (c$\mathrm{t}-\mathrm{c}$ ) pencil-beam distances were varied between $1 \mathrm{~mm}$ and $8 \mathrm{~mm}$ in a diagonal lattice pattern (Fig. 1). We have verified in an initial study that this diagonal square lattice offers better target volume coverage than the upright square lattice microbeam pattern used by other authors [1-4]. Therefore the c-t-c distance quoted in this work refers to lateral beam separation. To obtain the closest pencil beam separation distance for a diagonal lattice, our c-t-c value
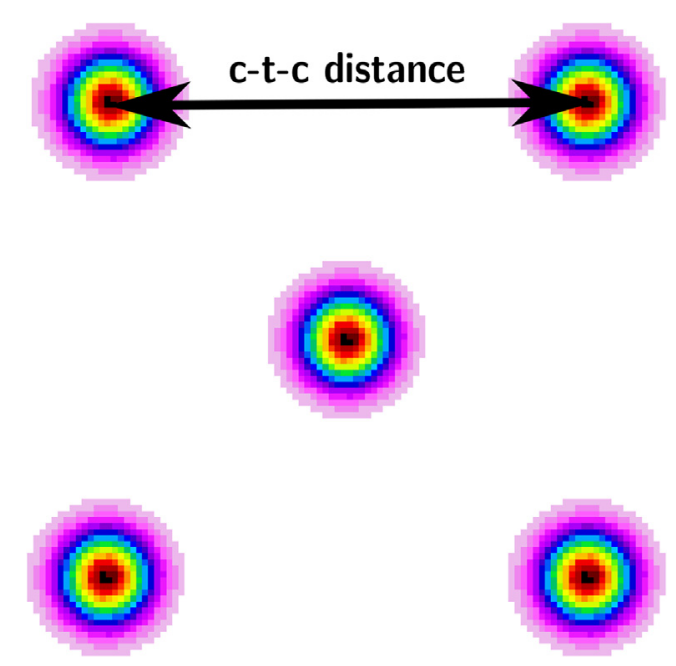

Figure 1. The proton microbeam diagonal square lattice irradiation pattern, indicating the definition of lateral centre-to centre (c-t-c) distances used in this work. An entrance irradiation field of $2 \times 2 \mathrm{~cm}^{2}$ was composed of microbeams arranged according to this pattern, with c-t-c distances ranging between 1 and $8 \mathrm{~mm}$. 
should be multiplied by the ratio $\sqrt{2} / 2$. The microbeam beam axes were parallel to each other and to the axis of the cylinder. The field size of $2 \times 2 \mathrm{~cm}^{2}$ was selected to simulate irradiation of a relatively small tumour inside this phantom.

To study the effect of proton scattering due to an outer bone layer (representing the skull bone) in the phantom, calculations were also performed in the same phantom to which a $0.5 \mathrm{~cm}$ thick layer of compact bone (based on ICRU tissue composition, as given by the FLUKA material data base [9]), was added, as shown in Fig. 2. To maintain the same beam ranges inside such a phantom, the beam entrance energies were increased to $63.5,83.0,102.6$ and $122.3 \mathrm{MeV}$, respectively. Again, calculations were then performed in this phantom, for c-t-c distances, ranging between $1 \mathrm{~mm}$ and $8 \mathrm{~mm}$.

In our PVDR calculations, local dose was defined as the sum of energy deposition events within voxels of a predefined size $(10 \mu \mathrm{m} \times 500 \mu \mathrm{m}$, in horizontal and longitudinal beam directions, respectively). While recognising that choice of the third (vertical) dimension of this voxel may strongly influence PVDR values calculated along the beam depth, for reasons of symmetry, we always took the vertical voxel dimension to be one-half of the current c-t-c distance (e.g., $500 \mu \mathrm{m}$ being one-half of the $1 \mathrm{~mm}$ c-t-c distance). The effect of selection of $250 \mu \mathrm{m}$ or $500 \mu \mathrm{m}$ as the vertical voxel dimension on the calculated PVDR values with depth, for a $60 \mathrm{MeV}$ proton beam calculation with c-t-c distance of $1 \mathrm{~mm}$, is shown in Table 1. The calculated dose in each voxel was normalised to the dose per one incident proton. To extract the PVDR values, cross profiles were calculated at steps of $5 \mathrm{~mm}$ depth in the phantom, in voxels of aforementioned sizes.

We described the homogeneity of dose deposition in the target volume (at the depth of the Bragg peak or within the range of the spread-out Bragg peak) by the ratio between the minimum dose, $D_{\min }$, and maximum dose $D_{\max }$, (expressed in percent), choosing $D_{\min } / D_{\max }>95 \%$ as the criterion of homogeneity, to reflect the spirit of ICRU recommendations $[10,11]$. We tested this criterion over the central $1 \times 1 \mathrm{~cm}^{2}$ part of the $2 \times 2 \mathrm{~cm}^{2}$ field used in our calculations.

To further study the microchannel concept in an extended target volume over the range of the SOBP, we simulated a suitable composition of proton beams of energies between 40 and $60 \mathrm{MeV}$ in steps of $2 \mathrm{MeV}$ (for the "bone phantom": 43.5-63.5 MeV, respectively) with a $1 \mathrm{~mm}$ lateral c-t-c distance between the pencil beams. Local and global dose distributions were compared with that calculated for the Bragg peak produced by a $60 \mathrm{MeV}$ microchannel proton beam.

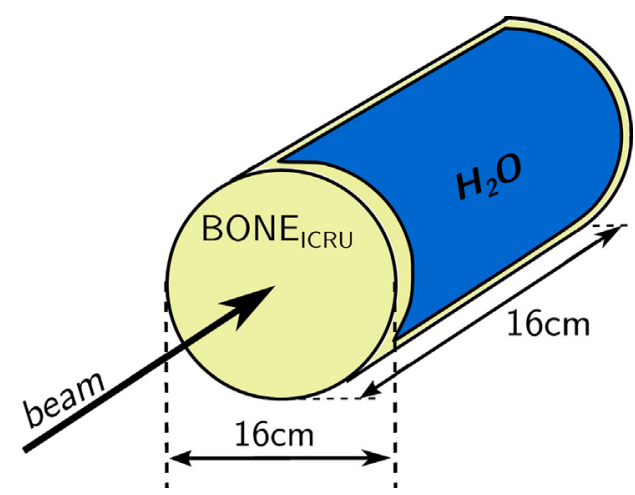

Figure 2. Design of head phantoms used in this work: in the water-bone phantom, a $5 \mathrm{~mm}$ thick outer layer of ICRU bone composition was added without changing the dimensions of the water phantom.
Table 1

Values of peak-to-valley dose ratios (PVDR) calculated for a $60 \mathrm{MeV}$ proton microbeam configuration with c-t-c distance of $1 \mathrm{~mm}$, calculated at different depths using vertical voxel sizes of $250 \mu \mathrm{m}$ or $500 \mu \mathrm{m}$. In further calculations, for reasons of symmetry, vertical voxel sizes of one-half of the c-t-c distances were always used (see text).

\begin{tabular}{crrrrr}
\hline Depth in phantom $[\mathrm{mm}]$ & \multicolumn{2}{c}{ Water phantom } & & \multicolumn{2}{c}{ Water-bone phantom } \\
\cline { 2 - 3 } \cline { 6 - 6 } & \multicolumn{1}{c}{$250 \mu \mathrm{m}$} & $500 \mu \mathrm{m}$ & & $250 \mu \mathrm{m}$ & $500 \mu \mathrm{m}$ \\
\hline 5.0 & $140.7 \pm 9.2$ & $32.0 \pm 0.8$ & & $77.7 \pm 3.8$ & $20.8 \pm 0.5$ \\
10.0 & $19.0 \pm 0.5$ & $7.2 \pm 0.1$ & & $6.0 \pm 0.1$ & $3.4 \pm 0.1$ \\
15.0 & $2.4 \pm 0.1$ & $1.8 \pm 0.1$ & & $1.3 \pm 0.1$ & $1.2 \pm 0.1$ \\
20.0 & $1.1 \pm 0.1$ & $1.0 \pm 0.1$ & & $1.0 \pm 0.1$ & $1.0 \pm 0.1$ \\
25.0 & $1.0 \pm 0.1$ & $1.0 \pm 0.1$ & & $1.0 \pm 0.1$ & $1.0 \pm 0.1$ \\
\hline
\end{tabular}

\section{Results and discussion}

To exemplify the particular aspects of the microbeam irradiation technique we note that, in the case e.g. of $60 \mathrm{MeV}$ pencil proton microbeams ( $1 \sigma=100 \mathrm{um}$ ) entering the medium (water) with a ct-c distance of $1 \mathrm{~mm}, 86 \%$ of these protons will travel in narrow channels of diameter $4 \sigma(0.4 \mathrm{~mm})$, which constitute about $22 \%$ of the entrance plane. If the c-t-c distance is increased to $2 \mathrm{~mm}$ according to the pattern shown in Fig. 1, the percentage of the irradiated volume at this plane decreases to $5.4 \%$. Consequently, the fraction of irradiated skin cells will decrease with increasing c-t-c distances and fluences (and corresponding local doses) across the entrance field within each micro-channel will be higher by reciprocals of these percentages.

We wished first to study the conditions at which transverse homogeneity may be achieved at target depth, i.e. at the depth of the Bragg peak (BP). Figure 3 shows the result of a systematic calculation of uniformity of dose coverage at respective BP depths in the water phantom for beams of energies ranging from 60 to $120 \mathrm{MeV}$, and c-t-c distances from 1 to $8 \mathrm{~mm}$. To obtain homogeneity better than $90 \%$ at the highest considered beam energy $(120 \mathrm{MeV})$, the c-t-c distances should not exceed $6 \mathrm{~mm}$. At $100 \mathrm{MeV}$ beam energy somewhat poorer homogeneity can still be achieved for c-t-c values ranging between 3 and $5 \mathrm{~mm}$, and at $80 \mathrm{MeV}$ - for $\mathrm{c}-\mathrm{t}-\mathrm{c}$ values of $3 \mathrm{~mm}$ or less. In the case of the lowest beam energy (60 MeV), it is possible to fulfil the $95 \%$ homogeneity criterion at BP

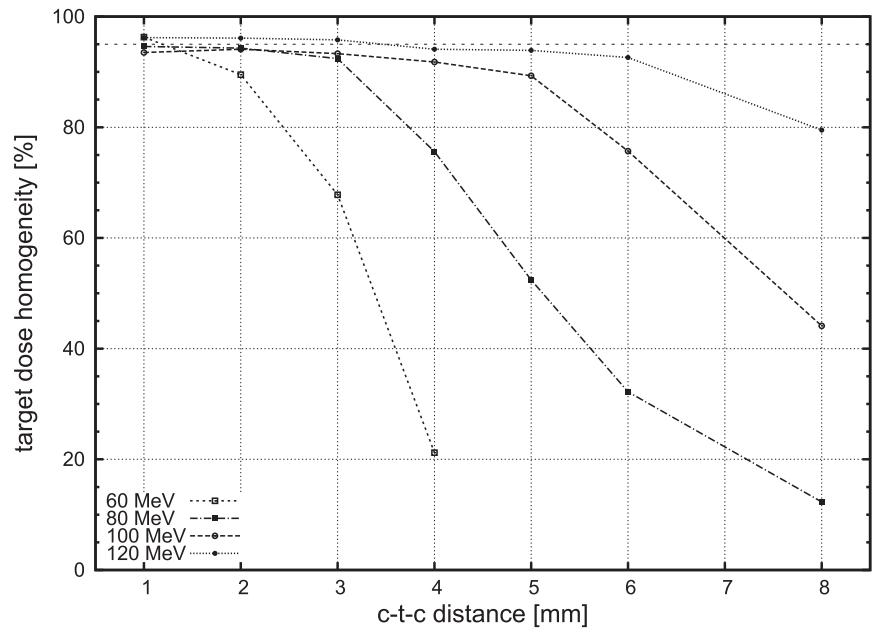

Figure 3. Field dose homogeneity at depths of corresponding Bragg peaks, after irradiation of the water-only phantom with proton microbeams $(\sigma=100 \mu \mathrm{m})$ of energy $60 \mathrm{MeV}, 80 \mathrm{MeV}, 100 \mathrm{MeV}$ and $120 \mathrm{MeV}$, with c-t-c spacing ranging between 1 and $8 \mathrm{~mm}$. The 95\% reference level is shown by a dotted line. For clarity, uncertainties of dose calculation $( \pm 2 \%)$ are not shown. 
depth for $1 \mathrm{~mm}$ c-t-c distance, as demonstrated in Fig. 4. These results illustrate the ranges of $\mathrm{c}-\mathrm{t}-\mathrm{c}$ distances and of proton energies for which uniform dose distribution in the target may be achieved.

In similar calculations performed for the water phantom with bone layers, dose uniformity was generally improved by some $4 \%$ in most situations, due to higher beam scattering by the $5 \mathrm{~mm}$-thick entrance bone layer.

Following earlier reports on photon microbeam irradiations using synchrotron radiation [1,2], we have assumed that protection of healthy tissues depends on the spatial distribution of the peakto-valley dose ratio, PVDR, which characterizes the nonuniformity between the irradiated and non-irradiated regions. We therefore calculated the PVDR values in proton microbeams for $\mathrm{c}-\mathrm{t}$-c distances where reasonable dose homogeneity could be achieved at target (BP) depths. As outlined above, we recognise the dependence of the calculated PVDR value on the voxel volume ( $c f$. Table 1), also noting that the calculated value of PVDR does not scale with voxel volume and depends on depth in phantom (as shown in Table 1, at larger depths the result is the same for either voxel volume). Over the entrance region, the smaller is the voxel volume, the higher is the calculated PVDR value. This is to be expected, as most of the beam energy is deposited close to its path.

In Table 2 we show results of systematic calculations of PVDR values at different depths, for beams of entrance energies 60, 80, 100 or $120 \mathrm{MeV}$ in the water-alone or water-with-bone head phantoms, for c-t-c distances between 1 and $6 \mathrm{~mm}$. In general, in the water phantom, after a depth of $5 \mathrm{~mm}$ the PVDR values decrease fivefold, while in the water-with-bone phantom they decrease by a factor of 10 . At greater depths the gradual decrease in PVDR values is roughly by a factor of two per $5 \mathrm{~mm}$ step in depth, for either phantom. In Fig. 4 we illustrate the strong dependence of PVDR values on c-t-c distances (range: $1-4 \mathrm{~mm}$ ) at a BP depth of $30 \mathrm{~mm}$ for $60 \mathrm{MeV}$ microbeams, in the water phantom. At this depth, reasonable conformity is obtained with $1 \mathrm{~mm}$ c-t-c distance.

Scattering of protons in the microbeams is enhanced as the atomic number $\mathrm{Z}$ of absorber increases, and is less pronounced as their energy decreases. These effects lead to a broadening of the initially narrow beam channels with depth in tissue, to a depth at which PVDR approaches unity, being absorber material-dependent. This may be seen in Fig. 5, where we show the calculated depthdose distribution of relative absorbed dose in the head phantom, over steps of $5 \mathrm{~mm}$ in depth. Addition of a $5 \mathrm{~mm}$-thick ICRU bone

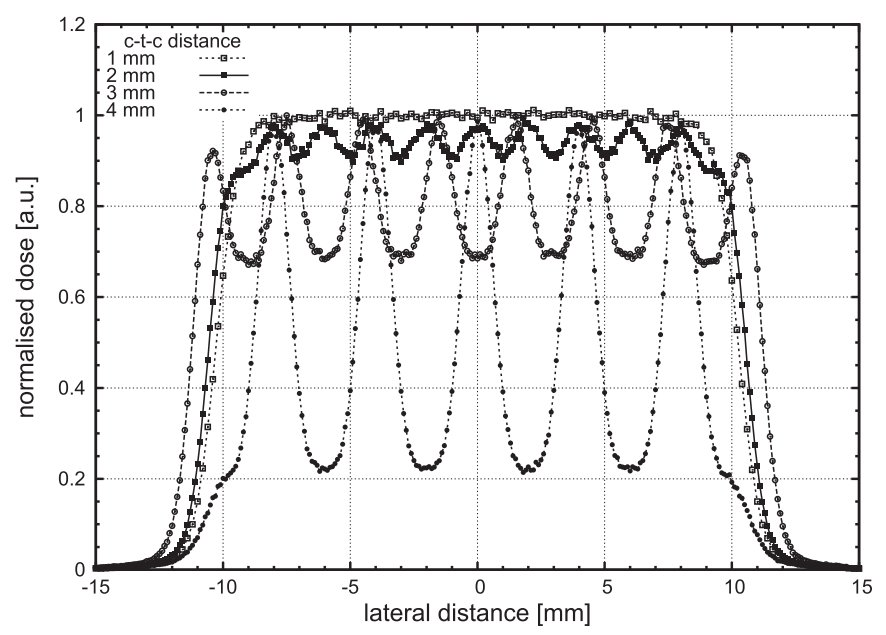

Figure 4. Transverse dose distributions of $60 \mathrm{MeV}$ proton microbeams $(\sigma=100 \mu \mathrm{m})$ with c-t-c distances of 1-4 mm, at $30 \mathrm{~mm}$ depth in the water phantom, normalised to their maximum values. For clarity, uncertainties of dose calculation $( \pm 2 \%)$ are not shown. layer decreases the initial PVDR by some 30\% due to additional beam spreading by the bone material which is denser than water. In the background of this figure we plotted the total dose distribution integrated over the entire phantom volume.

We found the entrance values of PVDR at the phantom surface to be relatively high (between 70 and 7300) and to depend on the proton entrance energy. Since protons of higher energy produce more energetic delta-rays, their radial range expands, hence their corresponding PVDRs at entrance are lower if the same detector voxel volumes are considered. However, PVDR values for slower protons decrease more rapidly at increasing depths in the phantom because of enhanced proton scattering as their energy decreases. The effect of c-t-c distance variation is therefore highly dependent on the entrance beam energy, acting only to a depth of about $10 \mathrm{~mm}$ for a $60 \mathrm{MeV}$ microbeam set up with a $1 \mathrm{~mm}$ lateral c-t-c distance, and to a depth of some $50 \mathrm{~mm}$ for a $120 \mathrm{MeV}$ microbeam with a c-t-c distance of $6 \mathrm{~mm}$, at which the PVDR value is about 10 .

In proton radiotherapy the lateral beam penumbra, $\mathrm{P}_{90 / 10}$, characterizes the steepness of the edges of transverse dose distribution profiles at given depths, between $90 \%$ and $10 \%$ dose levels. In Fig. 6 we show dose profiles extracted at BP depths for a set of microbeams delivering a $2 \times 2 \mathrm{~cm}^{2}$ entrance field to the water-bone phantom, where the $P_{90 / 10}$ values increase from less than $2.5 \mathrm{~mm}$ for a $63.5 \mathrm{MeV}$ microbeam (c-t-c distance of $1 \mathrm{~mm}$ ) to $6 \mathrm{~mm}$ for a 122.3 $\mathrm{MeV}$ one (c-t-c distance of $6 \mathrm{~mm}$ ). In the water phantom, the field flatness and the lateral penumbrae are not very different, at respective entrance microbeam energies and distances (results not shown). Clearly, for such small target volumes any proton microbeam profile cannot compete with the sharp penumbrae of photon MRT beams [1,2] as, in tissue, $100 \mathrm{keV}$ X-rays are mainly absorbed rather than scattered.

To evaluate PVDR distributions at different depths in a spreadout Bragg peak configuration, we performed a rough SOBP calculation for proton beams with adjusted fluence weighting. The entrance energies of these beams, with c-t-c separation of $1 \mathrm{~mm}$, ranged between 40 and $60 \mathrm{MeV}$ in $2 \mathrm{MeV}$ steps. We aimed at achieving SOBP modulation of $1.6 \mathrm{~cm}$, and simulated a $1 \times 1 \mathrm{~cm}^{2}$ field in the water or water-bone phantoms. The result is illustrated in Fig. 7. This microbeam separation distance was sufficient to achieve conformal and uniform dose coverage of the distal target region in a $60 \mathrm{MeV}$ proton beam. At lower beam energies, however, the $1 \mathrm{~mm}$ c-t-c distance was too large to achieve uniformity, resulting in considerable dose variation within the target volume (up to 15\%). Thus, in principle, SOBP microbeam irradiation is feasible, also for multiple fields [4]. However, achieving uniform dose distribution within the SOBP of proton microbeams requires a trade-off between larger centre-to-centre distances of the pencil beams (resulting in higher PVDR values at larger depths) and dose uniformity within the treatment volume. As the latter also strongly depends on the beam range, a sophisticated tool would need to be developed to achieve an acceptable optimised SOBP treatment plan.

The biological processes underlying microbeam radiotherapy are not well understood. The sparing effect of microscopic beams in normal tissue is believed to be connected with rapid regeneration of microvessels in the irradiated tissue cells outside the microbeam paths (i.e. in the "valleys" of the transversal dose distributions), hence the importance of the PVDR) [1,2]. It is not clear to what extent the increasing values of dose at the "valleys" with beam depths observed in our proton microbeam simulations are biologically significant. Another mechanism to consider is microbeam damage to the network of microvessels surrounding the tumour which could lead to tumour tissue necrosis due to loss of blood perfusion. The response of the microvasculature of normal tissues and that of the tumour to high local doses may differ, the latter 
Table 2

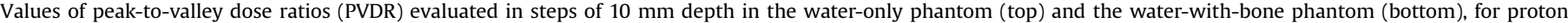
microbeams $(\sigma=100 \mu \mathrm{m})$ of different entrance energy and c-t-c distances. Empty entries in these tables represent PVDR values of 1 till the corresponding BP depth.

\begin{tabular}{|c|c|c|c|c|c|}
\hline & \multirow[t]{2}{*}{ Depth in phantom [mm] } & \multicolumn{4}{|c|}{ Centre-to-centre distance } \\
\hline & & $1 \mathrm{~mm}$ & $3 \mathrm{~mm}$ & $5 \mathrm{~mm}$ & $6 \mathrm{~mm}$ \\
\hline \multirow[t]{11}{*}{ Water phantom } & & $60 \mathrm{MeV}$ & $80 \mathrm{MeV}$ & $100 \mathrm{MeV}$ & $120 \mathrm{MeV}$ \\
\hline & 5.0 & $32.0 \pm 0.9$ & $480.6 \pm 34.6$ & $1019.6 \pm 20.7$ & $1229.7 \pm 49.3$ \\
\hline & 15.0 & $1.8 \pm 0.1$ & $98.1 \pm 6.0$ & $267.4 \pm 16.9$ & $372.9 \pm 11.5$ \\
\hline & 25.0 & $1.0 \pm 0.1$ & $14.6 \pm 0.4$ & $92.7 \pm 5.9$ & $155.4 \pm 7.1$ \\
\hline & 35.0 & & $2.6 \pm 0.1$ & $30.3 \pm 1.2$ & $70.8 \pm 1.8$ \\
\hline & 45.0 & & $1.1 \pm 0.1$ & $8.4 \pm 0.3$ & $28.9 \pm 1.2$ \\
\hline & 55.0 & & & $2.9 \pm 0.1$ & $10.5 \pm 0.3$ \\
\hline & 65.0 & & & $1.4 \pm 0.1$ & $4.3 \pm 0.1$ \\
\hline & 75.0 & & & $1.0 \pm 0.1$ & $2.2 \pm 0.1$ \\
\hline & 85.0 & & & & $1.4 \pm 0.1$ \\
\hline & 95.0 & & & & $1.1 \pm 0.1$ \\
\hline \multirow[t]{11}{*}{ Water-bone phantom } & & 63.5 MeV & 83.0 MeV & $102.6 \mathrm{MeV}$ & $122.3 \mathrm{MeV}$ \\
\hline & 5.0 & $20.8 \pm 0.5$ & $346.6 \pm 22.4$ & $781.1 \pm 37.8$ & $916.2 \pm 9.7$ \\
\hline & 15.0 & $1.2 \pm 0.1$ & $57.7 \pm 3.7$ & $178.9 \pm 11.9$ & $268.8 \pm 3.5$ \\
\hline & 25.0 & $1.0 \pm 0.1$ & $7.3 \pm 0.2$ & $63.2 \pm 3.1$ & $112.7 \pm 6.3$ \\
\hline & 35.0 & & $1.8 \pm 0.1$ & $18.2 \pm 0.6$ & $50.3 \pm 1.6$ \\
\hline & 45.0 & & $1.0 \pm 0.1$ & $5.4 \pm 0.1$ & $18.8 \pm 0.6$ \\
\hline & 55.0 & & & $2.2 \pm 0.1$ & $7.1 \pm 0.1$ \\
\hline & 65.0 & & & $1.2 \pm 0.1$ & $3.2 \pm 0.1$ \\
\hline & 75.0 & & & $1.0 \pm 0.1$ & $1.8 \pm 0.1$ \\
\hline & 85.0 & & & & $1.2 \pm 0.1$ \\
\hline & 95.0 & & & & $1.0 \pm 0.1$ \\
\hline
\end{tabular}

perhaps being more vulnerable to microbeam damage, explaining the enhanced therapeutic effect of the microbeams [3]. The effect of the microbeam on the skin may also be related to the size of the irradiated area and the ionization density of the microbeam radiation, as observed via skin erythema or ulceration of pig skin irradiated by small sources [12].

It is not clear to what extent our results obtained for proton MRT can be interpreted via photon MRT investigations [1,2]. According to our PVDR calculations, any benefit from the peak-and-valley shape of the lateral dose distribution would be effective only over the first $25-35 \mathrm{~mm}$ in depth, for proton microbeams of entrance energies ranging between 60 and $120 \mathrm{MeV}$. Beyond those depths, we should expect no difference between standard proton therapy and the micro-beam method as the dose distribution is relatively

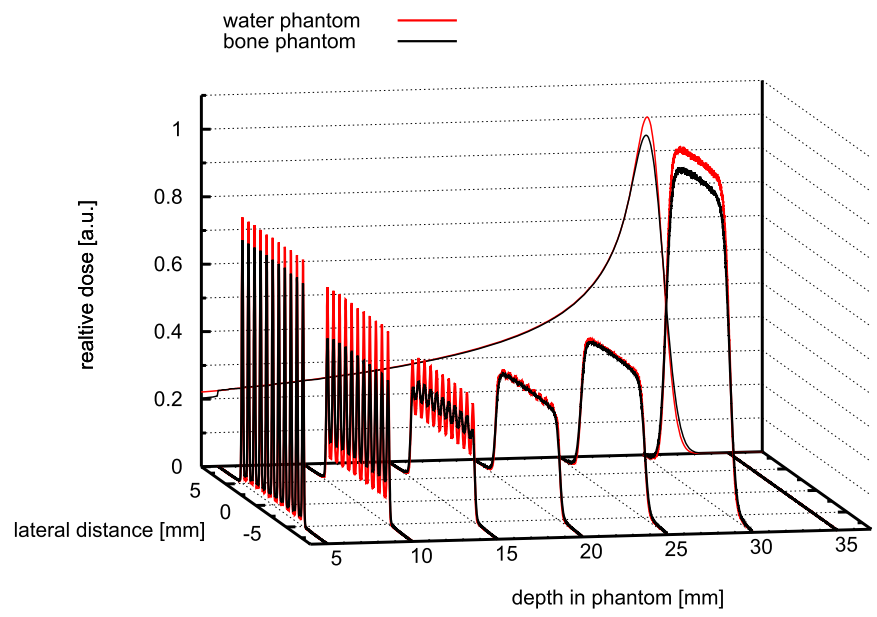

Figure 5. Depth dose distributions for a set of proton microbeams ( $\sigma=100 \mu \mathrm{m}$, c-t$\mathrm{c}=1 \mathrm{~mm}$ ) forming a $1 \times 1 \mathrm{~cm}^{2}$ entrance field for a $60 \mathrm{MeV}$ beam entering axially the water-only (red) or water-with-bone (black) head phantoms. These dose distributions are normalised to their maxima at BP depth. In the background the total dose integrated over the entire phantom volume is plotted. For clarity, uncertainties of dose calculation $( \pm 2 \%)$ are not shown. (For interpretation of the references to colour in this figure legend, the reader is referred to the web version of this article.) uniform. When considering skull irradiation in children, where a high entrance dose could lead to undesired late effects (such as bone malformation), in the proton microbeam approach one could perhaps decrease the irradiated volume by limiting the spot number and increasing the spot distances. Also, further optimisation of the number and entrance energies of microbeam ports could be considered. Such a complex approach to beam optimisation will not, however, be possible without relevant radiobiological data which could address the effect of the entrance dose reduction and tumour response in proton MRT. Application of a well optimized irradiation scheme, in terms of spot size and distribution, entrance beam energy, or number of ports, combined with such radiobiological investigations, could then pave the way for

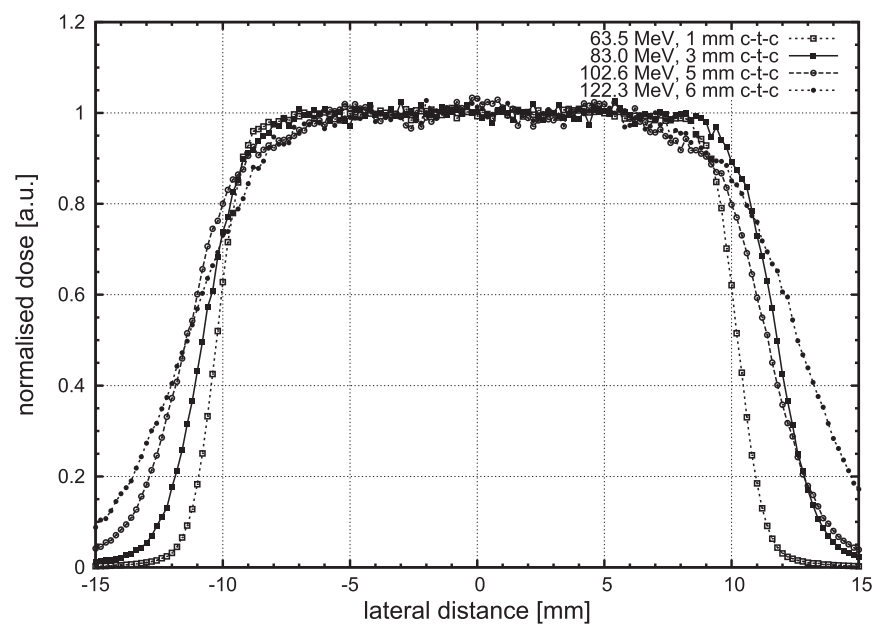

Figure 6. Lateral dose profiles in the water-with-bone phantom for a set of proton microbeams $\left(\sigma=100 \mu \mathrm{m}\right.$ ) forming a $2 \times 2 \mathrm{~cm}^{2}$ entrance field, calculated for different beam entrance energies and c-t-c separation, at respective Bragg peak depths in phantom: $30 \mathrm{~mm}$ (63.5 MeV, c-t-c $1 \mathrm{~mm}), 51 \mathrm{~mm}(83.0 \mathrm{MeV}, \mathrm{c}-\mathrm{t}-\mathrm{c} 3 \mathrm{~mm}), 76,5 \mathrm{~mm}$ (102.6 MeV, c-t-c $5 \mathrm{~mm}$ ) and $105.5 \mathrm{~mm}(122.3 \mathrm{MeV}$, c-t-c $6 \mathrm{~mm})$. Profiles are normalised to their maximum values. For clarity, uncertainties of dose calculation $( \pm 2 \%)$ are not shown. 


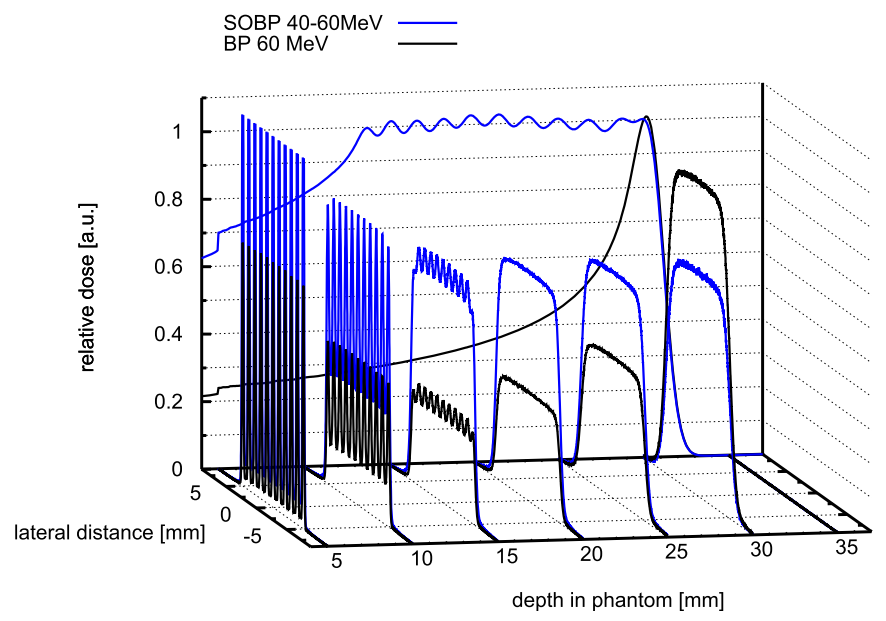

Figure 7. Depth dose distributions in the water-with-bone head phantom for a set of proton microbeams ( $\sigma=100 \mu \mathrm{m}, \mathrm{c}-\mathrm{t}-\mathrm{c}=1 \mathrm{~mm}$ ) forming a $1 \times 1 \mathrm{~cm}^{2}$ entrance field in a spread-out Bragg peak (SOBP) configuration (blue), and for a pristine pencil beam of $63.5 \mathrm{MeV}$ entrance energy (black). Each distribution is normalised to its respective value at BP depth. The SOBP configuration was designed to cover a target volume of $1 \times 1 \times 1.6 \mathrm{~cm}^{3}$ with proton beams of maximum energy $63.5 \mathrm{MeV}$. For beams of lower energies dose homogeneity inside the SOBP region falls below the $95 \%$ level (e.g., it is $85 \%$ at $15 \mathrm{~mm}$ depth). In the background the total dose integrated over the entire phantom volume is plotted. For clarity, uncertainties of dose calculation $( \pm 2 \%)$ are not shown. (For interpretation of the references to colour in this figure legend, the reader is referred to the web version of this article.)

hypofractionation scenarios where lower entrance dose, better malignant tissue response and better conformity to the target volume could be achieved, compared with the more common proton beam radiotherapy procedures.

\section{Conclusions}

Microbeam radiation therapy offers potential benefit from highdose collimated micro-size photon layers by increasing healthy tissue recovery together with better local tumour control. The proposed technique of proton microbeam radiotherapy combines the advantages of small beam size with proton scattering for target dose escalation. Active scanning technique could then be used with a pre-calculated map of spots to take into account the contribution from the adjacent pencil-beams. The obtained PVDR values are comparable with photon MRT data over depths of some 15-25 mm in tissue, especially for protons of entrance energies above $80 \mathrm{MeV}$ [3]. The additional advantage of the proton MRT is in the well-defined proton range, which is of particular importance, e.g. in brain radiotherapy. We showed the possibility of beam optimization by taking into account the beam size, c-t-c distance or entrance energy of the beam. By combining several ports one could significantly boost the absorbed dose in the target volume possibly arriving at a superior method for high-precision PVDRmode irradiation, especially for hypofractionation scenarios. None of these expectations can, however, be fulfilled without better understanding of the involved biology and radiobiology of both healthy and malignant tissues irradiated by proton microbeams, nor without the considerable engineering effort to develop innovative techniques of delivering such beams.

\section{Acknowledgements}

This research was supported in part by PL-Grid Infrastructure. We gratefully acknowledge the help of Dr. Urszula Jeleń of UKGM Marburg, Germany, in implementing the SOURCE routine and of Dr. Leszek Grzanka of IFJ PAN Kraków, Poland, in supplying preliminary data for SOBP calculations. This work was performed within the Marian Smoluchowski Cracow Scientific Consortium - KNOW funding programme. The COST Action TD1205 (SYRA3) is gratefully acknowledged for the received support.

\section{References}

[1] Brauer-Krisch E, Serduc R, Siegbahn EA, Le Duc G, Prezado Y, Bravin A, et al. Effects of pulsed, spatially fractionated, microscopic synchrotron X-ray beams on normal and tumoral brain tissue. Mutat Res Rev Mutat Res 2010;704: 160-6.

[2] Dilmanian FA, Qu Y, Liu S, Cool CD, Gilbert J, Hainfeld JF, et al. X-ray microbeams: Tumor therapy and central nervous system. Nuclear Instrum Methods Phys Res Sec A 2005;548(1-2):30-7.

[3] Prezado Y, Fois GR. Proton-minibeam radiation therapy: a proof of concept. Med Phys 2013;40(3)

[4] Zlobinskaya O, Girst S, Greubel Ch, Halbe V, Siebenwirth Ch, Walsh DWM, et al. Reduced side effects by proton microchannel radiotherapy: study in a human skin model. Radiat Environ Biophys 2013;52:123-33.

[5] Datzmann G, Dollinger G, Goeden C, Hauptner A, Körner H-J, Reichart P, et al. The Munich microprobe SNAKE: first results using $20 \mathrm{MeV}$ protons and 90 MeV sulfur ions. Nuc Inst Methods Phys Res Sec B. Beam Interact Mater Atoms $2001 ; 181(1-4): 20-6$.

[6] Ferrari A, Sala PR, Fassò A, Ranft J. FLUKA: a multi-particle transport code, CERN-2005-10. 2005. INFN/TC_05/11, SLAC-R-773.

[7] Bohlen TT, Cerutti F, Chin MPW, Fassò A, Ferrari A, Ortega PG, et al. The FLUKA code: developments and challenges for high energy and medical applications. Nuclear Data Sheets 2014;120:211-4.

[8] Slatkin DN, Spanne P, Dilmanian FA, Sandborg M. Micorbeam radiation tharepy. Med Phys 1992;19:1295-400.

[9] ICRU Report 37. Stopping powers for electrons and positrons. In: International commission on radiation units and Measurements; 1984.

[10] ICRU Report 62. Prescribing, recording and reporting photon beam therapy. In: International Commission on Radiation Units and Measurements; 1999.

[11] ICRU Report 78. Prescribing, recording and reporting proton beam therapy. In: International Commission on Radiation Units and Measurements; 2007.

[12] Lippincott SW, Wilson JD, Montour JL. Radiation effects on pig skin. Exposure to different densities of ionization. Arch Pathol 1975 Feb;2:105-10. 